\title{
Microscopic Irreversibility: Looking for a Microscopic Description of Time Asymmetry
}

\author{
Oliver Penrose ${ }^{1}$
}

Received: 3 February 2020 / Accepted: 7 April 2020 / Published online: 4 May 2020

(c) The Author(s) 2020

\begin{abstract}
This paper is an attempt to understand time-reversal asymmetry better by developing the quantitative description of that asymmetry. The aim is not to explain the asymmetry, but to describe it in more detail. Two model systems are considered here; one is the classical Lorentz gas, the other a quantum Lorentz gas. In the classical case, it is argued that the distribution of the directions of motion of particles that are about to hit an obstacle is qualitatitvely different from the analogous distribution for particles that have just hit the obstacle (an entropy-like functional of the velocity distribution function is used to characterize the asymmetry). In the quantum case, a similar distinction is drawn between the density matrix describing particles that have not yet encountered an obstacle and the one describing particles that have hit an obstacle or are in the process of doing so.
\end{abstract}

Keywords Irreversibility $\cdot$ Lorentz gas $\cdot$ Convex functions $\cdot H$-theorem

\section{Introduction}

Everybody knows that the real world has a time direction : that is to say, its macroscopic behaviour is not symmetrical under time reversal. Yet that world consists of particles and waves whose motion, at the microscopic level of description, is believed to satisfy differential equations ("laws") which are symmetrical under time reversal. Much has been written about the difficulty of reconciling these two types of description. The difficulty is often called the irreversibility paradox.

William Thomson, whose name later on changed to Lord Kelvin, was one of the first to discuss this difficulty. Writing [1] in 1874 he contrasted what he called "abstract dynamics",

This paper is dedicated to Joel Lebowitz on the occasion of his 90th birthday, in celebration of over 64 years of friendship

Communicated by Ivan Corwin.

Oliver Penrose

O.Penrose@ma.hw.ac.uk

1 Department of Mathematics, Maxwell Institute for Mathematical Sciences, Heriot-Watt University, Riccarton, Edinburgh EH14 4AS, Scotland, UK 
meaning the mathematical equations decribing the motion of individual molecules, which are symmetrical under time reversal, with "physical dynamics", which includes frictional forces and is not reversible. To illustrate the paradox he wrote:

"If, then, the motion of every particle of matter in the universe were precisely reversed at any instant the course of nature would be simply reversed for ever after. The bursting bubble of foam at the foot of a waterfall would reunite and descend into the water, the thermal motions would reconcentrate their energy and throw the mass up the fall in drops re-forming into a close column of ascending water. Heat which had been generated by the friction of solids and dissipated by conduction would come again to the place of contact, and throw the moving body back against the force to which it had previously yielded. Boulders would recover from the mud the materials required to rebuild them into their previous jagged forms, and would become reunited to the mountain peak from which they had formerly broken away".

Discussion of this paradox often centres on the question how to explain the irreversible behaviour of matter, given that the molecules it is made of satisfy reversible equations. ${ }^{1}$ In other words, given that certain motions such as the cascade in a waterfall actually occur, how is it that the reversed motions, which are also compatible with the microscopic equations of motion, do not also occur? If an answer to this question is given it usually involves some plausible assumption about the initial conditions of the motion, but such explanations leave open the further question of why one can reasonably make such assumptions about the initial conditions but not about the final conditions.

The aim of the present paper is less ambitious - not to try to explain anything, merely to describe it. This aim is consistent with the way things have often happened before : scientists first describe some aspect of the way real things behave and only afterwards find a good way to explain it. For example, in 1605 Kepler observed that planetary orbits could be accurately described as ellipses, and this made possible Newton's later (1687) explanation of these elliptical shapes in terms of his universal law of gravitation. Similarly, if one wished to explain why it is that our hearts are on the left side, a first step might be to listen to the chests of a lot of people, and to investigate which other creatures also have hearts on the left side, before trying to explain why Nature chose this particular asymmetry. The description of time asymmetry to be given here does not claim to explain anything at all, but rather it is offered in the hope that it may help others to explain, or at least clarify, the relation between the time-reversal symmetry of the microscopic dynamical laws and the usual lack of such symmetry in the behaviour of the real world.

In physics, there is one exceptional "law" that is not a differential equation and is not symmetrical under time reversal : the Second Law of thermodynamics. This law states that if no heat enters or leaves a macroscopic system, then its entropy cannot decrease. In particular, if the system is isolated, its entropy cannot decrease. As Boltzmann [6] pointed out in his reply to Loschmidt's criticism [7] of his recently published kinetic equation, this "law" differs from the others in being statistical in character. That is, the Second Law does not say that the increase of entropy is required by the laws of mechanics and therefore a mathematical certainty; only that this increase is extremely probable, i.e. that it is a practical certainty for the motions that actually occur.

The idea of the present paper is to put forward a principle which, like the second law of thermodynamics, is statistical in character, but which is much more detailed, giving information not just about the macroscopic evolution of macroscopic systems but rather about the motion of individual atoms. The suggested principle is a plausible microscopic statistical property of a system of interacting particles, which is not symmetric under time reversal.

${ }^{1}$ See, for example, Spohn [2] or Lebowitz [3,4] or Goldstein [5] 


\section{A Lorentz gas}

To set out the main idea in a reasonably simple context, let us consider a much-studied model system, the Lorentz gas [8] (sometimes called the wind-tree model). This model consists of a gas of point particles which do not interact with each other but make elastic collisions with a collection of fixed smooth hard scatterers.

Every time a particle hits a scatterer it arrives travelling in a straight line, and on bouncing off the scatterer it departs travelling along a different straight line. We denote the velocity of arrival by $\mathbf{u}$, the velocity of departure by $\mathbf{v}$, and a unit vector (the normal) in the direction of the line from the centre of the scatterer to the point of impact by $\mathbf{n}$ (see Figs. 1, 2).

Since the scatterers are smooth and elastic, the speeds of arrival and departure are equal:

$$
|\mathbf{u}|=|\mathbf{v}|
$$

and the angles of incidence and of reflection are also equal. The direction of the normal vector $\mathbf{n}$ bisects the angle between the direction $-\mathbf{u}$ (note the minus sign) from which the particle came before the collision and the direction $\mathbf{v}$ towards which it will go after the collision, so that

$$
\mathbf{n}=((-\mathbf{u})+\mathbf{v}) \times \text { positive scalar }
$$

\section{Notation for the Two-Dimensional Case}

For simplicity all the calculations in this paper will be done for the two-dimensional case. We take the polar coordinates of the vectors $-\mathbf{u}, \mathbf{v}, \mathbf{n}$ to be

$$
-\mathbf{u}=(|\mathbf{u}|, \theta), \quad \mathbf{v}=(|\mathbf{v}|, \phi) \quad \mathbf{n}=(1, v)
$$

The algebraic relation between these three angles depends on the magnitude $|\theta-\phi|$. In the case illustrated in Fig. 1, this magnitude is less than $\pi$, and the relation is

$$
v=\frac{1}{2}(\theta+\phi) \text { if }|\theta-\phi|<\pi
$$

In the case illustrated in Fig. 2, where $|\theta-\phi|>\pi$, the corresponding formula is

$$
\nu=\frac{1}{2}(\theta+\phi) \pm \pi \quad \text { if }|\theta-\phi|>\pi
$$

where the choice between + and - is made so that

$$
|v|<\pi
$$

The angle of incidence (which equals the angle of reflection) is half the angle between the directions of the vectors $\mathbf{u}$ and $\mathbf{v}$. A formula for the (signed) angle of incidence, denoted here by $\chi$, is

$$
\begin{aligned}
& \chi=\frac{1}{2}(\theta-\phi) \quad \text { if }|\theta-\phi|<\pi \quad \text { (see Fig. 1) } \\
& \text { but }=\frac{1}{2}(\theta-\phi) \pm \pi \text { if }|\theta-\phi|>\pi \quad \text { (see Fig. 2) }
\end{aligned}
$$

the choice between + and - in Eq. (8) being made so that

$$
|\chi|<\frac{1}{2} \pi
$$




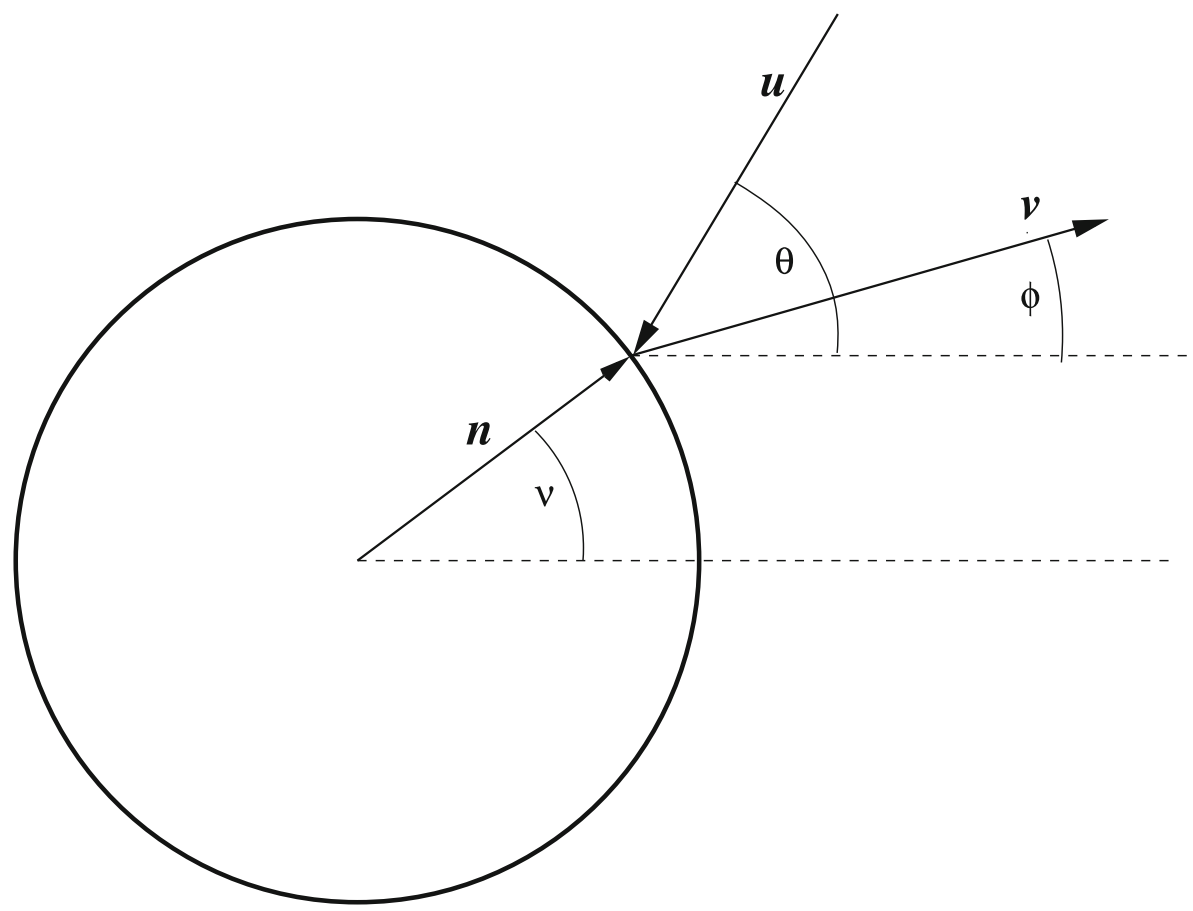

Fig. 1 The particle approaches the scatterer with velocity $\mathbf{u}$ and recedes with velocity $\mathbf{v}$. The normal vector $\mathbf{n}$ is parallel to $(-\mathbf{u})+\mathbf{v}$. The angle between the vector $-\mathbf{u}$ and the positive $x$ axis is $\theta$; the angle between $\mathbf{v}$ and the positive $x$ axis is $\phi$. This diagram illustrates the case where $|\theta-\phi|<\pi$, so that the angle between the vectors $-\mathbf{u}$ and $\mathbf{v}$ is less than $\pi$. The polar angle $v$ of the normal vector $\mathbf{n}$ is equal to $(\theta+\phi) / 2$

\section{A Statistical Description}

Let us assume that the density of the Lorentz gas is large enough to justify describing it statistically, i.e. by using a distribution function rather than by giving the trajectory of each individual particle. We assume that the distribution function is changing slowly in comparison with the mean free time, so that to a first approximation it can be treated as independent of time. Then we can think of the distribution of particle states as a time-independent probability distribution ${ }^{2}$ of trajectories in two-dimensional space.

We concentrate on a particular scatterer and consider the particles that hit it during a particular time interval, short in comparison with the mean free time. To study the timereversal asymmetry of the probability distribution of trajectories, we shall compare two joint probability densities: the joint probability density of the pre-collision random variables $-\mathbf{u}$ and $\mathbf{n}$, which we denote by $f(-\mathbf{u}, \mathbf{n})$ or $f(|\mathbf{u}|, \theta, v)$, and the joint probability density of the post-collision variables $\mathbf{v}$ and $\mathbf{n}$, which we denote by $g(\mathbf{v}, \mathbf{n})$ or $g(|\mathbf{v}|, \phi, v)$.

Since the random variables $\mathbf{u}, \mathbf{v}, \mathbf{n}$ are connected by the relation (4) or (5), their probability densities are also related. Indeed, it follows from (4) or (5), and the consequent fact that the Jacobian of the mapping $(\theta, v) \rightarrow(\phi, v)$ has modulus 1 , that the probability densities $f$ and

${ }^{2}$ N.B. This probability distribution is not connected with any Gibbs ensemble; it is to be interpreted as an approximate "broad-brush" way of describing a single many-particle system, in the spirit of Maxwell and Boltzmann 


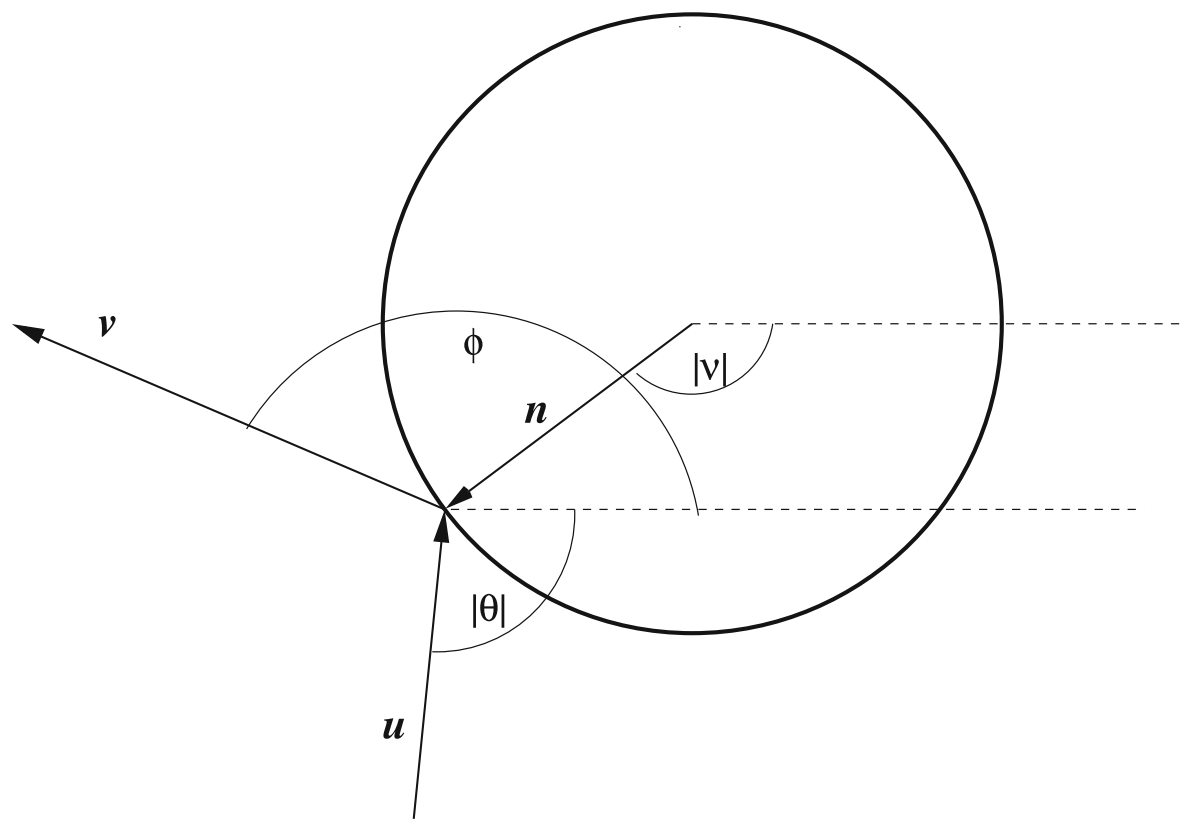

Fig. 2 An illustration of what can happen when $|\theta-\phi|>\pi$. In the case shown, $\theta<-\frac{1}{2} \pi$ and $\phi>\frac{1}{2} \pi$. The normal vector $\mathbf{n}$ is in the opposite direction to $\mathbf{v}+(-\mathbf{u})$; its polar angle $v$ is $v=(\phi+\theta) / 2-\pi$ The numbers $\theta$ and $\nu$, representing angles in the third quadrant, are both negative, so that $\theta=-|\theta|$ and $\nu=-|\nu|$

$g$ are related by

$$
f(|\mathbf{u}|, \theta, v)=g(|\mathbf{u}|, 2 v-\theta, v),
$$

where, if necessary, we define the function $g(|\mathbf{u}|, \phi, \nu)$ outside the range $-\pi<\phi<\pi$ by requiring it to be periodic in $\phi$ with period $2 \pi$.

Under the time reversal transformation

$$
\mathbf{u} \rightarrow-\mathbf{v}, \mathbf{v} \rightarrow-\mathbf{u}, \theta \rightarrow \phi, \phi \rightarrow \theta
$$

the angle $v$ is invariant and the formula (10) maps to

$$
f(|\mathbf{v}|, \phi, v)=g(|\mathbf{v}|, 2 v-\phi, v),
$$

which, as a relation between the functions $f$ and $g$, is equivalent to (10).

However, it will be argued here that, despite this time-reversal symmetry of the relation (10) connecting the two functions $f$ and $g$, the functions themselves are likely to be significantly different.

\section{A Time-Asymmetric Conjecture}

Let us examine the consequences of a plausible conjecture about the particle trajectories, namely that the directions of the incoming particles are statistically independent of the places where they hit the scatterer. That is to say, in two dimensions, if a particle comes in from some given direction $\theta$, we are conjecturing that the the angle of incidence at the collision, namely $\chi$, is independent of the direction $\theta$ from which the particles are coming. In other words, the 
incoming particles form a collection of beams, each of which is wider than the diameter of the scatterer, and in each of which all the particles are travelling in exactly the same direction. This is an approximation, since in fact each of the incoming 'beams' of particles does not come from infinitely far away but emanates from a previous scatterer, at a distance which has the same order of magnitude as the mean free path; the approximation corresponds to taking the mean free path to be much greater than the diameter of a scatterer.

According to this independence conjecture, the joint probability density of the incoming velocity $(|\mathbf{u}|, \theta)$ and the angle of incidence $\chi$ is a product of two factors, one being the distribution of velocities describing the collection of incoming beams and the other being the probability density (in $\chi$ space) that a given incoming particle will hit the scatterer at a place where the angle of incidence is $\chi$.

Since the latter factor (normalized) is $\frac{1}{2} \cos \chi$, and the various angles are related by (4) or (5) and (7) or (8) so that $\chi=\theta-v$ or $\chi=\theta-v \pm \pi$, the product distribution is

$$
f(|\mathbf{u}|, \theta, v)=h(|\mathbf{u}|, \theta) \frac{1}{2}|\cos (\theta-v)|
$$

where $h(|\mathbf{u}|, \theta)$ is the velocity distribution of the incoming beams.

Using Eq. (10) with the notation change $\theta \rightarrow 2 v-\phi$, and then (13), we find that

$$
\begin{aligned}
g(|\mathbf{v}|, \phi, v) & =f(|\mathbf{v}|, 2 v-\phi, v) \\
& =h(|\mathbf{v}|, 2 v-\phi) \frac{1}{2}|\cos (v-\phi)|
\end{aligned}
$$

If the system is not in equilibrium, the distribution of incoming particles will in general be non-uniform, and the two probability densities $f$ and $g$ will be different. The nature of this difference can be clarified by looking at the marginal distributions of the velocities of the incoming and outgoing particles. For the incoming particles the marginal distribution of velocities, denoted here by $F$, is

$$
\begin{aligned}
F(|\mathbf{u}|, \theta) & :=\int_{\theta-\pi / 2}^{\theta+\pi / 2} \mathrm{~d} v f(|\mathbf{u}|, \theta, v) \\
& =\int_{\theta-\pi / 2}^{\theta+\pi / 2} \mathrm{~d} v h(|\mathbf{u}|, \theta) \frac{1}{2}|\cos (\theta-v)| \\
& =h(|\mathbf{u}|, \theta)
\end{aligned}
$$

For the outgoing particles, the marginal distribution of velocities, denoted here by $G$, is

$$
\begin{aligned}
G(|\mathbf{v}|, \phi) & :=\int_{\phi-\pi / 2}^{\phi+\pi / 2} \mathrm{~d} v g(|\mathbf{v}|, \phi, v) \\
& =\int_{\phi-\pi / 2}^{\phi+\pi / 2} \mathrm{~d} v h(|\mathbf{v}|, 2 v-\phi) \frac{1}{2}|\cos (v-\phi)| \quad \text { by }(14) \\
& =\int_{-\pi}^{\pi} \mathrm{d} \theta h(|\mathbf{v}|, \theta) \frac{1}{2}\left|\cos \left(\frac{\theta-\phi}{2}\right)\right| \quad(\text { where } \theta:=2 v-\phi) \\
& =\int_{-\pi}^{\pi} \mathrm{d} \theta F(|\mathbf{v}|, \theta) \frac{1}{2}\left|\cos \left(\frac{\theta-\phi}{2}\right)\right| \text { by (15) }
\end{aligned}
$$

Equation (16) shows that (at constant $|\mathbf{v}|$ ) the function $G(|\mathbf{v}|, \cdot)$ is the convolution of the function $F(|\mathbf{v}|, \cdot)$ with the normalized non-negative function $\left|\frac{1}{2} \cos \frac{1}{2}(\cdot)\right|$ 


\section{A Convexity Inequality}

Let $\Psi(\cdot)$ be any convex function of a real variable (i.e. one whose graph does not go below any tangent). Then, since the kernel of the convolution in (16) is a normalized probability density, Jensen's inequality [9] tells us that, for each value of $\phi$,

$$
\Psi\left(\int_{-\pi}^{\pi} \frac{1}{2}\left|\cos \left(\frac{\phi-\theta}{2}\right)\right| h(|\mathbf{v}|, \theta) \mathrm{d} \theta\right) \leq \int_{-\pi}^{\pi} \frac{1}{2}\left|\cos \left(\frac{\phi-\theta}{2}\right)\right| \Psi(h(|\mathbf{v}|, \theta)) \mathrm{d} \theta
$$

(for more explanation, see the Appendix). Using (15) and (16), Eq. (17) can be written

$$
\Psi(G(|\mathbf{v}|, \phi)) \leq \int_{-\pi}^{\pi} \frac{1}{2}\left|\cos \left(\frac{\phi-\theta}{2}\right)\right| \Psi(F(|\mathbf{v}|, \theta)) \mathrm{d} \theta
$$

Integrating over $\phi$ and using the normalization property of the function $\frac{1}{2} \cos \frac{1}{2}(\cdot)$ we get

$$
\begin{aligned}
\int_{-\pi}^{\pi} \Psi(G(|\mathbf{v}|, \phi)) \mathrm{d} \phi & \leq \int_{-\pi}^{\pi}\left(\int_{-\pi}^{\pi} \frac{1}{2}\left|\cos \left(\frac{\phi-\theta}{2}\right)\right| \mathrm{d} \phi\right) \Psi(F(|\mathbf{v}|, \theta)) \mathrm{d} \theta \\
& =\int_{-\pi}^{\pi} \Psi(F(|\mathbf{v}|, \theta)) \mathrm{d} \theta
\end{aligned}
$$

Thus the left side, which refers to the distribution just after collision, is less than the right side which refers to the distribution just before collision and therefore refers to a slightly earlier time than the left side.

The proposition put forward in this paper is that the time-asymmetric inequality

$$
\int \Psi(G) \mathrm{d} \phi \leq \int \Psi(F) \mathrm{d} \theta
$$

is not restricted to the special case where it was derived, but rather that it holds in a large variety of cases and could be the basis for a general distinction between past and future in terms of microscopic quantities.

\section{Time Evolution and an "H-Theorem"}

Imagine a Lorentz gas in which the positions of the scatterers are chosen at random in accordance with a probability distribution that is uniform in space. At time $t=0$ the particles are assumed to be distributed uniformly in space, with their velocities distributed independently of each others' and of their positions. The distribution of the particles' velocities at time 0 will be denoted (using polar coordinates) by $F_{0}(|\mathbf{v}|, \phi)$. Moreover, since a particle's velocity changes only when it hits a scatterer, at any time after $t=0$ the distribution of velocities of those particles that have not yet hit any scatterers will still be $F_{0}$.

In Sect. 5 we denoted the distribution of the directions of motion of particles that are just about to hit a scatterer by $F$. For the situation considered in the present section, our definitions imply that the function $F$ immediately after time 0 is given by

$$
F(|\mathbf{v}|, \phi)=F_{0}(|\mathbf{v}|, \phi)
$$

Now consider the particles that have hit exactly one scatterer since time 0 , denoting the distribution of their velocities by $F_{1}$. The work in Sect. 5 shows that

$$
G(|\mathbf{v}|, \phi)=F_{1}(|\mathbf{v}|, \phi)
$$


Then, assuming the truth of the proposition (20), it follows that

$$
\int_{-\pi}^{\pi} \Psi\left(F_{1}\right) \mathrm{d} \phi \leq \int_{-\pi}^{\pi} \Psi\left(F_{0}\right) \mathrm{d} \phi
$$

where $\Psi(\cdot)$ is any convex function.

More generally, let us denote by $F_{n}$ the distribution of velocities of particles that have made exactly $n$ collisions since time 0 . Then, assuming that the proposition (20) applies to the $n$th collision, the result (23) generalizes to

$$
\int_{-\pi}^{\pi} \Psi\left(F_{n+1}\right) \mathrm{d} \phi \leq \int_{-\pi}^{\pi} \Psi\left(F_{n}\right) \mathrm{d} \phi
$$

Since $n$ increases monotonically with time, Eq. (24) tells us that the quantity

$$
H(t,|\mathbf{v}|):=\int_{-\pi}^{\pi} \Psi(F(t,|\mathbf{v}|, \phi)) \mathrm{d} \phi,
$$

where $F(t,|\mathbf{v}|, \phi)$ denotes the velocity distribution $F(|\mathbf{v}|, \phi)$ at time $t$, is a non-increasing function of the time $t$ for each value of the speed $|\mathbf{v}|$. Thus our proposition (20) leads to an " $H$-theorem" similar to Boltzmann's $H$-theorem in the kinetic theory of gases $[6,10]$; his theorem also asserts that a quantity $H$ defined by a formula similar to (25) is a non-increasing function of time. The $H$ in Boltzmann's theorem is defined by choosing $\Psi(x):=x \log x$ in equation (25) and integrating over velocity space, and its non-increase property follows from Boltzmann's kinetic equation for $F$. There is a similar " $H$-theorem" in the theory of the low-density Lorentz gas, where the kinetic equation for $F$ is called the linear Boltzmann equation [10-12], and the convex function $\Psi(x)$ is taken to be $x^{2}$.

Boltzmann's derivation of his kinetic equation, like our derivation of the monotonic increase result (25), made use of a time-asymmetric assumption, the molecular chaos assumption or Stosszahlansatz [13], which is applied separately at each collision. Our proposition (20) can be thought of as a generalization of the molecular chaos assumption which, it is hoped, will hold in more general situations than the one considered in its derivation, for example at high densities.

\section{The Quantum-Mechanical Case}

Can a similar idea be used in quantum mechanics? The quantum mechanical case is conceptually more complicated; for example it is not in general possible to say with certainty where a particle is, so that one cannot say with certainty whether or not it has collided with a particular scatterer. Nevertheless ...

In Sect. 4 of this paper we made the approximation of treating the particles approaching a given scatterer as a mixture of beams. The corresponding approximation in quantum mechanics would be to treat the incoming particles as a mixture of plane waves. Each plane wave can be labelled by its wave vector $\mathbf{k}$ so that its wave function $\psi(\mathbf{x})$ is proportional to $\mathbf{e}^{i \mathbf{k} \cdot \mathbf{x}}$. In analogy with the classical case (see Sect. 4), we take the probability distribution of the plane wave states to be $F(\mathbf{k}) \mathrm{d}^{2} \mathbf{k}$ with the normalization

$$
\int \mathrm{d}^{2} \mathbf{k} F(\mathbf{k})=1
$$


In the position representation, the density matrix of this mixture of plane waves is

$$
\left\langle\mathbf{x}^{\prime}\left|D^{(0)}\right| \mathbf{x}^{\prime \prime}\right\rangle=\rho \int \mathrm{d}^{2} \mathbf{k} F(\mathbf{k}) \mathbf{e}^{i \mathbf{k} \cdot\left(\mathbf{x}^{\prime}-\mathbf{x}^{\prime \prime}\right)}
$$

where $\rho$ is the number of particles per unit area and the proportionality constant has been chosen (using (26)) so that the diagonal elements of the density matrix in position representation are equal to $\rho$. In the wave-number (momentum) representation, the density matrix is

$$
\left\langle\mathbf{k}^{\prime}\left|D^{(0)}\right| \mathbf{k}^{\prime \prime}\right\rangle=4 \pi^{2} \rho F\left(\mathbf{k}^{\prime}\right) \delta\left(\mathbf{k}^{\prime}-\mathbf{k}^{\prime \prime}\right)
$$

The statistical operator $D^{(0)}$ is a reasonable model for describing the gas a long way from any scatterer, but it does not take into account the interaction of the gas particles with the scatterers. The scatterers affect the motion of the particles in a way that may reasonably be modelled, like any other time evolution, by a unitary transformation. This unitary transformation will be denoted here by $U$, so that the statistical operator after an interaction with the scatterers has the form

$$
D=U D^{(0)} U^{-1}
$$

In the wave-number representation Eq. (29) gives, using (28) and the unitary property of $U$,

$$
\begin{aligned}
\left\langle\mathbf{k}^{\prime}|D| \mathbf{k}^{\prime \prime}\right\rangle & =4 \pi^{2} \rho \iint\left\langle\mathbf{k}^{\prime}|U| \mathbf{k}^{\prime \prime \prime}\right\rangle \mathrm{d}^{2} \mathbf{k}^{\prime \prime \prime} F\left(\mathbf{k}^{\prime \prime \prime}\right) \delta\left(\mathbf{k}^{\prime \prime \prime}-\mathbf{k}^{\mathrm{iv}}\right) \mathrm{d}^{2} \mathbf{k}^{\mathrm{iv}}\left\langle\mathbf{k}^{\mathrm{iv}}\left|U^{-1}\right| \mathbf{k}^{\prime \prime}\right\rangle \\
& =4 \pi^{2} \rho \int\left\langle\mathbf{k}^{\prime}|U| \mathbf{k}^{\prime \prime \prime}\right\rangle F\left(\mathbf{k}^{\prime \prime \prime}\right)\left\langle\mathbf{k}^{\prime \prime}|U| \mathbf{k}^{\prime \prime \prime}\right\rangle \mathrm{d}^{2} \mathbf{k}^{\prime \prime \prime}
\end{aligned}
$$

The probability distribution of momenta after the interaction is given by the diagonal elements of this matrix, which by (30) are

$$
\rho G\left(\mathbf{k}^{\prime}\right)=\left(\mathbf{k}^{\prime}|D| \mathbf{k}^{\prime}\right)=4 \pi^{2} \rho \int\left|\left\langle\mathbf{k}^{\prime}|U| \mathbf{k}^{\prime \prime \prime}\right\rangle\right|^{2} F\left(\mathbf{k}^{\prime \prime \prime}\right) \mathrm{d}^{2} \mathbf{k}^{\prime \prime \prime}
$$

Thus the distributions of momenta before and after the interaction (or collision) are related by a convolution relation closely resembling the one (16) which we found for the classical Lorentz gas. And therefore, just as in the classical case, one can use Eq. (20) to give a numerical comparison between the "before" and "after" versions of the momentum distribution.

\section{Summary and Conclusions}

This paper draws attention to a simple time-asymmetric feature of the probability distributions describing the motion in a Lorentz gas. It is claimed that this asymmetric feature applies in quantum mechanics as well as classical. The asymmetry is expressed mathematically by an inequality (20) asserting that a negentropy-like functional of the distribution of velocities of the particles approaching a scatterer exceeds the corresponding quantity for the particles receding from that scatterer.

As an example, if the velocities of the incoming particles are all nearly the same, the velocities of the outgoing particles will (according to the inequality proposed here) vary much more widely. The plausibility of the asymmetric principle used here can be judged by considering the ridiculous implausibility of the time inverse of the motion just described. In the time reversed motion, particles would come in from many different directions with their trajectories so finely controlled that they would all bounce off the scatterer in approximately the same direction. 
Acknowledgements Grateful thanks are due to Joel Lebowitz, for countless discussions and arguments about interesting statistical mechanics matters including irreversibility_and to him and his wife Ann for a lifetime of friendship. I should also like to thank Paul Hare, Mathew Penrose, Herbert Spohn and the referee for helpful comments, and Derek Richards of the Open University, UK, for asking me (roughly 40 years ago) an extremely good question, to which neither of us had any answer at the time, but it set me thinking : is there a general way to characterize irreversible behaviour in a large (or even medium sized) quantum system?.

Open Access This article is licensed under a Creative Commons Attribution 4.0 International License, which permits use, sharing, adaptation, distribution and reproduction in any medium or format, as long as you give appropriate credit to the original author(s) and the source, provide a link to the Creative Commons licence, and indicate if changes were made. The images or other third party material in this article are included in the article's Creative Commons licence, unless indicated otherwise in a credit line to the material. If material is not included in the article's Creative Commons licence and your intended use is not permitted by statutory regulation or exceeds the permitted use, you will need to obtain permission directly from the copyright holder. To view a copy of this licence, visit http://creativecommons.org/licenses/by/4.0/.

\section{Appendix: Expanded Derivation of Eq. (20)}

Let us write Eq. (16) of the main text in the simplified notation

$$
G(\phi)=\int_{-\pi}^{\pi} k(\phi, \theta) F(\theta)
$$

where

$$
k(\phi, \theta):=\frac{1}{2}\left|\cos \frac{1}{2}(\theta-\phi)\right|
$$

is a probability distribution, satisfying for each value of $\phi$ the normalization condition

$$
\int_{-\pi}^{\pi} \mathrm{d} \phi k(\phi, \theta)=1
$$

To say that the function $\Psi$ is convex means that for each $x_{0}$ in the domain of $\Psi$ there exists a 'slope' $\Psi^{\prime}\left(x_{0}\right)$ such that the graph of the function $\Psi$ lies above or upon the tangent at $x_{0}$, i.e.

$$
\Psi(x) \geq \Psi\left(x_{0}\right)+\left(x-x_{0}\right) \Psi^{\prime}\left(x_{0}\right) \quad \forall x \forall x_{0}
$$

For each value of the variable $\phi$ and each function $f$ on the domain $(-\pi, \pi)$ let us define the expectation

$$
E_{\phi}(f):=\int_{-\pi}^{\pi} k(\phi, \theta) f(\theta) \mathrm{d} \theta \quad \forall \phi
$$

so that, by (32) and (34),

$$
\begin{aligned}
& E_{\phi}(F)=G(\phi) \\
& E_{\phi}(G)=G(\phi)
\end{aligned}
$$

In Eq. (35) we set $x=F(\theta)$ and $x_{0}=E_{\phi}(F(\theta))=G(\phi)$, obtaining

$$
\Psi(F(\theta)) \geq \Psi(G(\phi))+(F(\theta)-G(\phi)) \Psi^{\prime}(G(\phi))
$$

Applying the linear functional $E_{\phi}$ defined in (36) to each term of this equation, regarded as a function of $\theta$ at fixed $\phi$, we get

$$
E_{\phi}(\Psi(F(\theta))) \geq E_{\phi}(\Psi(G(\phi)))+\left(E_{\phi}(F(\theta))-G(\phi)\right) \Psi^{\prime}(G(\phi))
$$




$$
\begin{aligned}
& =\Psi(G(\phi))+(G(\phi)-G(\phi)) \Psi^{\prime}(G(\phi)) \text { by (37) and (38) } \\
& =\Psi(G(\phi))
\end{aligned}
$$

so that, using (36) again,

$$
\int_{-\pi}^{\pi} k(\phi, \theta) \Psi(F(\theta)) \mathrm{d} \theta \geq \Psi(G(\phi))
$$

Integrating with respect to $\phi$ and using (34) gives

$$
\int_{-\pi}^{\pi} \mathrm{d} \theta \Psi(F(\theta)) \geq \int_{-\pi}^{\pi} \mathrm{d} \phi \Psi(G(\phi))
$$

which is Eq. (19) of the main text.

\section{References}

1. Thomson, W.: The kinetic theory of the dissipation of energy. Proc. R. Soc. Edinb. 8, 325 (1874). reprinted in Brush (1966) Kinetic Theory, vol 2, (Pergamon, Oxford, 1966); see also the shorter version Kinetic Theory of the Dissipation of Energy, Nature, April 9, 1874, pp. 441-444

2. Spohn, H.: Large Scale Dynamics of Interacting Particles, p. 49 and Chapter 9. Springer (1991)

3. Lebowitz, J.L.: Macroscopic laws, microscopic dynamics, time's arrow and Boltzmann's entropy. Physica A 194, 1-27 (1993). https://doi.org/10.1016/0378-4371(93)90336-3

4. Lebowitz, J.L.: Boltzmann's entropy and time's arrow. Phys. Today 46(9), 32 (1993). https://doi.org/10. $1063 / 1.881363$

5. Goldstein, S.: Boltzmann's Approach to Statistical Mechanics, pp 39-54 of Chance in Physics, Foundations and Perspectives. In: Bricmont, J., Dürr, D., Galavotti, M.C., Ghirardi, G., Petruccione, F., Zanghi, N. (eds.). Springer (2001). https://www.springer.com/gp/book/9783540420569

6. Boltzmann, L.: p. 59 of Lectures on gas theory by Stephen G. Brush (Univ. of California Press, Berkeley and Los Angeles 1964) which is the English translation of Boltzmann, L.: Vorlesungen über Gastheorie, J. A. Barth, Leipzig 1896 (1898)

7. Loschmidt, J.: Wien. Ber. 73, 128, 366 (1876), for further early references see page 58 of the previous reference

8. Lorentz, H.: Le mouvement des électrons dans les métaux. Arch. Néerl. 10, 336-371 (1905)

9. Jensen, J.L.W.V.: Sur les fonctions convexes et les inégalités entre les valeurs moyennes. Acta Math. 30(1), 175-193 (1906). https://doi.org/10.1007/BF02418571

10. Cercignani, C.: Theory and Application of the Boltzmann Equation, pp. 172-173. Springer, Edinburgh and London (1975)

11. Gallavotti, G.: Rigorous theory of the Boltzmann equation in the Lorentz gas, Nota Interna No. 358, Istituto di Fisica, Universita di Roma. https://www.citeseerx.ist.psu.edu/newdoc/summary (1972)

12. Spohn, H.: The Lorentz process converges to a random flight process. Commun. Math. Phys 60, 277-290 (1978)

13. Burbury, S.H.: Nature 5178 (1894) and 52104 (1895), cited by S. G. Brush in The kind of motion we call heat in Studies in Statistical Mechanics, ed. E W Montroll and J L Lebowitz vol VI (North Holland, Amsterdam (1976)

Publisher's Note Springer Nature remains neutral with regard to jurisdictional claims in published maps and institutional affiliations. 